sons, and another great service would be done by a less comprehensive book which dealt with the Comparative Method at an introductory level and was more accessible to biologists from the experimental fields. Within Harvey's Department at Oxford are also some leading developmental biologists. When they and the evolutionary biologists start talking to each other we can expect some real progress.

ANDREW LEIGH BROWN
Centre for HIV Research
University of Edinburgh
West Mains Road
Edinburgh EH9 $3 J N$

\section{Genetic Disorders and the Fetus: Diagnosis, Prevention and Treatment (third edition). Edited by A. Milunsky. The Johns Hopkins University Press. 1992. 992 pages. $\$ 125.00$. ISBN 0801844134 .}

The original edition of 'Milunsky' appeared in 1979 , at the end of the first decade of the clinical application of prenatal diagnosis to chromosomal disorders, inborn errors of metabolism and congenital malformations. As a comprehensive summary of the state of the art it had few peers, and it became the reference text for genetic counsellors, obstetricians and laboratory personnel. More than half of the first edition was the personal work of Aubrey Milunsky, whose command of the literature was one of its most impressive features. In the second edition it became an edited work, and although this allowed expansion of topics covered, there was consequent loss of the unique Milunsky style.

The surprising feature of the third edition is how little different it is from the second, given the many achievements that have occurred in prenatal diagnosis over the last six years. It is even 15 pages shorter, for which some kind of ecological award should be made. Several chapters have been dropped - flow sorting of metaphase chromosomes, $\mathrm{Rh}$ disease and prenatal diagnosis and public policy. Two chapters have been added, on fetal cells in the maternal circulation and on fetal therapy. The main difference is that the sense that chapters had been thrown in the air and then ordered as they fell, which was such a startling aspect of the second edition, has gone. This is a logically organized book and now one has the feeling that Milunsky has the right formula and will stick with it. And so he should; this is a very successful book.

The main role that a reviewer should play in assessing the merits of a book of this nature is to rate it against the competition. Here I have a problem, because the only current alternative to Milunsky (M) is Prenatal Diagnosis and Screening, edited by Brock, Rodeck and Ferguson-Smith (BRF), and published by Churchill-Livingstone in 1992. Naturally I prefer the latter. I am tempted to do a Julie Burchill and tell you all about my book in this review of Milunsky, but instead I shall try a fair-minded comparison.
There are many similarities between the two, with $M$ weighing in at $3 \mathrm{lb}$ for its 880 pages and BRF at $6 \mathrm{lb}$ for its 785 . M costs $£ 94$ and BRF $£ 95$, the latter being printed on fine quality paper. $M$ gives you 29 chapters and 47 contributors, most of whom are American, while BRF has a more international distribution of 71 contributors over 47 chapters. Given the amazing time that it takes a publisher to produce a finished product from a manuscript, it is gratifying that both books managed to include the cloning of the fragile $\mathrm{X}$ gene (May 1991), and unsurprising that neither could cover the myotonic dystrophy gene (February 1992).

I do have one personal gripe about Milunsky's book. He invited me to contribute the chapter on cystic fibrosis, but when I pointed out that I was editing BRF and would be writing the chapter on CF in that, abruptly withdrew the invitation. What offends me is that my replacement has produced quite the most pathetic account of the subject $I$ have seen in a long time. Cystic fibrosis is one of the most important disorders for DNA based prenatal diagnosis and deserves better treatment.

The main difference between the two books lies in emphasis. $M$ is an encyclopaedic tome and probably cites every paper on prenatal diagnosis ever written. Occasionally, this makes it irritatingly uncritical, but as it is primarily aimed at people who want facts rather than opinions, this does not matter too much. The laboratory scientists will find it a hugely useful book. BRF is conscious that it is obstetricians who control access to prenatal diagnosis, and who because of the growing powers of imaging techniques, actually do the lion's share of the practice. Thus BRF has a whole section on congenital malformations and is particularly strong on ultrasonography. I would guess that if choice between the two has to be made, it is likely that it will be geneticists and scientists for $\mathbf{M}$ and obstetricians and other medical specialists for BRF.

DA VID BROCK

Human Genetics Unit University of Edinburgh

The Biology of Mosquitoes, Vol. 1: Development, Nutrition and Reproduction. By A. N. ClemENTS. Chapman \& Hall. 1992. 509 pages. Price $£ 69.00$. ISBN 0412401800 .

This book is an absolute must for any laboratory working on mosquitoes. It covers everything about their genetics, morphology and development as well as information on physiology and endocrinology.

It is a good mix of descriptive biology, essential for a handbook to be of value, and experimental studies. The diagrams and figures complement the text extremely well and the extensive reference list will be of great value to newcomers to particular areas of study.

Each chapter of the book is thoroughly researched and Alan Clements has gone to a great deal of trouble 
to consult researchers with expertise in particular areas to ensure all the sections are up to date.

One of the points I find particularly interesting is the tremendous diversity between mosquito species, there are enormous differences in the size of their genomes, their behaviour and in the pathogens and parasites they transmit. It seems to me that one of the biggest hurdles to controlling these disease vectors at present is our lack of understanding of the basic biology of the insects. Bringing all the information together in this way should help researchers determine where the gaps are and act accordingly.

MARY BOWNES University of Edinburgh Institute of Cell \& Molecular Biology Mayfield Road, Edinburgh EH9 3JR

Genome Analysis-Strategies for Physical Mapping. Vol. 4. Edited by Kay E. Davies and ShIRLEY M. Tilghman. Cold Spring Harbor Laboratory Press. 1992. 165 pages. Price \$49. ISBN 0879694122.

You really need to have considerable background knowledge about the progress and status of gene mapping in different organisms to appreciate the work described in the five chapters of this slim but demanding volume. The first four chapters wriggle along the evolutionary ladder from Escherichia coli to the pig (Sus scrofa), pausing on an uneven plateau of Drosophila melanogaster and Arabidopsis thaliana along the way. The plateau refers to the fact that Drosophila and Arabidopsis have comparable estimated haploid genome sizes of $165 \mathrm{Mb}$ and $100 \mathrm{Mb}$ respectively. Every schoolchild knows that Drosophila is virtually synonymous with genetic analysis, but Arabidopsis will not ring many bells. The status of the genome maps in these two organisms reflects this unevenness. The clear-cut organized progress in Drosophila is not surprising, given the biological resources available. However, it is heartening to see that integrated analysis of the Thale cress (Arabidopsis) has produced a very respectable map using visible traits, cloned gene markers and anonymous genomic clones in the form of YACs from several carefully produced and available libraries. The emphasis throughout for both of these organisms is the need to integrate the maps produced with the aid of different markers and place these onto the YAC contig maps which are the immediate aim for the fruitfly and the cress as well as for the more notorious human map which manages to escape much mention in this volume. Among the differences which are clearly emphasized for Drosophila and Arabidopsis, is the fact that the Dipteran is uniquely endowed with the pinboard for the ultimate in cytogenetic maps - the giant polytene salivary gland chromosomes - while Arabidopsis has hopelessly small chromosomes not amenable to mapping for example by in situ hybridization even to the extent that mammalian chromo- somes are. The pig map is in its earliest stages. Cytogenetic and contig mapping are possibilities shimmering on the horizon, but there is not yet a single marker assigned to three of the eighteen autosomes. However, although the possibility of genetic analysis is still being created by the systematic production of $\mathrm{F} 2$ hybrids between distant strains with a good number of allelic differences between them, it is clear to authors and readers alike that, given the necessary financial resources, the scientific tools are available for very rapid progress in creating a physical map onto which the genetic map can be superimposed and which will then permit direct selection of quantitative trait loci which are of major interest for a species whose main significance is commercial breeding.

In many ways the most disappointing chapter was the one describing the status of what should have been the best characterized organism: $E$. coli with a mere $4.6 \mathrm{Mb}$ genome. The problems of computer representation for a five-enzyme restriction map of this well-known bug engender despair in those of us who look forward to having graphical representation available soon for the whole human genome, where the average size of a single chromosome is thirty times that of the $E$. coli circle. Even the integration of the well-studied physical and genetic conjugation maps has not been achieved satisfactorily for $E$. coli.

The final chapter is an optimistic attempt at analysing how much individual YAC clones, particularly in the context of their contiguous neighbours, will be able to contribute to the complete definition of complex genomes. This area is extremely fast-moving especially in human genome analysis. The next critical step at least for the human map will be the more or less exhaustive identification and isolation of genes (accounting for only about $5 \%$ of total genomic DNA) from large genomic fragments. The methods for this are still in the pipeline, and a chapter in a book like this cannot hope to be up-to-date with details of the improved efficiency of exon-trapping mechanisms which recently made a great contribution to the isolation of the gene for Huntington's Disease, or the claim, at a meeting I have just attended, that a US team of 100 people has sequenced at least a portion of about a quarter of all expressed RNA molecules from the human genome. European input (French in the shape of CEPH and Genethon) into such factory-scale genome research has nearly covered the whole human genome with contiguous, if frequently chimaeric, YAC clones. The next improved library is already replacing these clones with cleaner, larger ones. Perhaps we shall see a complete human map before $E$. coli is fully finished. Certainly no paper-bound book can keep up with progress in the fast lane of physical mapping.

VERONICA VAN HEYNINGEN MRC Human Genetics Unit Edinburgh 